\title{
EXPERIMENTAL STUDIES OF SUPERSONIC RADIATIVELY COOLED PLASMA JETS
}

\author{
S.V. Lebedev ${ }^{1}$, F. Suzuki-Vidal ${ }^{1}$, M. Bocchi ${ }^{1}$, G.F. Swadling ${ }^{1}$, \\ G. Burdiak ${ }^{1}$, S.N. Bland ${ }^{1}$, P. de Grouchy ${ }^{1}$, A.J. Harvey-Thompson ${ }^{1}$, \\ E. Khoory ${ }^{1}$, S. Patankar ${ }^{1}$, L. Pickworth ${ }^{1}$, J. Skidmore ${ }^{1}$, R. Smith ${ }^{1}$, \\ J.P. Chittenden ${ }^{1}$, M. Krishnan ${ }^{2}$, R. Madden ${ }^{2}$, K. Wilson-Elliot ${ }^{2}$, \\ A. Ciardi ${ }^{3}$ and A. Frank ${ }^{4}$
}

\begin{abstract}
Properties of radiatively cooled supersonic plasma jets formed by ablation of thin Al foils driven by 1.4 MA, 250 ns current pulse are presented. The jets are highly collimated with half-opening angles of $\sim 2^{\circ}$. Measurements of the flow velocity $(\sim 60 \mathrm{~km} / \mathrm{s})$ and plasma temperature $(\sim 15 \mathrm{eV})$ in the jet with Thomson scattering diagnostic give internal Mach number of $\mathrm{M} \sim 3$, suggesting additional collimation of the jet by toroidal magnetic fields.
\end{abstract}

Appropriately scaled, high-energy density plasma experiments can complement our understanding of complex astrophysical phenomena (see Remington et al. 2006 for a review). Jets have been the subject of several studies addressing problems related to the launching and propagation (Bellan et al. 2005; Ciardi et al. 2007; Foster et al. 2005; Lebedev et al. 2005; Loupias et al. 2007). Here, we present laboratory experiments aiming to investigate interaction of supersonic, radiatively cooled jets with ambient medium. Scaling considerations require the dimensionless Reynolds (Re), magnetic Reynolds $\left(\operatorname{Re}_{M}\right)$, and Peclet (Pe) numbers to be much larger than unity; this implies that the transport of momentum, magnetic fields, and thermal energy, respectively, occurs predominantly through advection with the flow. For the experiments discussed here these conditions are satisfied and the typical values obtained are $\operatorname{Re} \sim 5 \times 10^{5}-10^{6}, \operatorname{Re}_{M} \sim 20$ and $\mathrm{Pe} \sim 100$.

\footnotetext{
${ }^{1}$ Imperial College London, London, UK

2 Alameda Applied Sciences Corporation, San Leandro, CA, USA

3 LERMA, Observatoire de Paris, École Normale Supérieure, Université Pierre et Marie Curie, CNRS, France

${ }^{4}$ University of Rochester, Rochester, NY, USA
} 
(a)

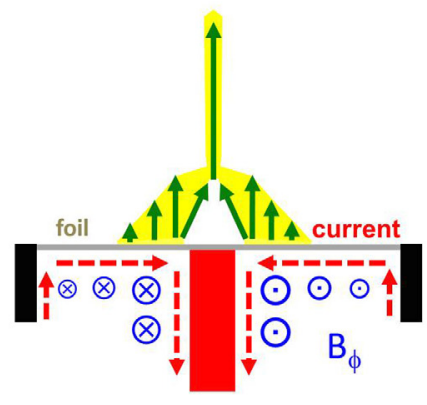

(b)

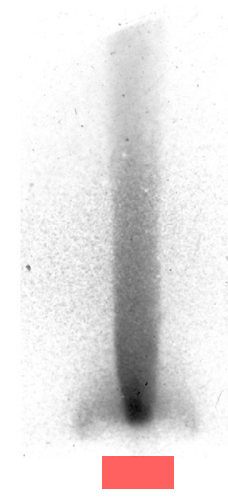

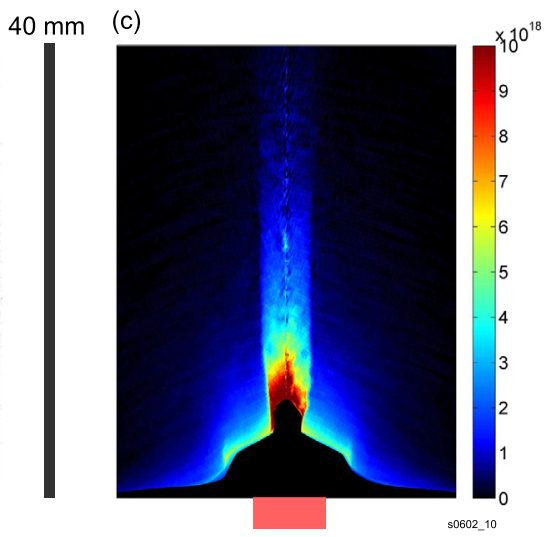

Fig. 1. Schematic of the experimental set-up (a), XUV images of the jet in vacuum (b) and electron density distribution measured by interferometry (c).

The jet in these experiments is produced using ablation of the surface of thin $\mathrm{Al}$ foil in a radial foil z-pinch configuration (Ciardi et al. 2009; Gourdain et al. 2010; Suzuki-Vidal et al. 2010) schematically shown in Figure 1a. Experiments are performed on the MAGPIE pulsed power facility, which provides a $1.4 \mathrm{MA}$, 250 ns current pulse through the foil. The morphology of a plasma jet in the absence of an ambient gas is illustrated by XUV emission image and by the map of electron density obtained from laser interferometry (Figs. 1b,c respectively). The jet is highly collimated and the flow is sustained for $\sim 450 \mathrm{~ns}$. The tip of the jet reaches a height of $\sim 35 \mathrm{~mm}$ above the foil, which corresponds to an aspect ratio of the jet (jet length/jet radius) exceeding $\sim 20$. The jet has a well defined smooth boundary and a high degree of collimation with a half-opening angle of $\sim 2^{\circ}$, and has no noticeable perturbations in its shape or in the intensity of the emission. Although the degree of jet collimation does not decrease with time, the measurements show a slow increase of the jet's diameter from $\sim 2.2 \mathrm{~mm}$ at $290 \mathrm{~ns}$ to $\sim 4.3 \mathrm{~mm}$ at $467 \mathrm{~ns}$ (at $\mathrm{z}=8 \mathrm{~mm}$ ), which corresponds to a characteristic radial expansion velocity of $\mathrm{V}_{R} \sim 5 \mathrm{~km} / \mathrm{s}$. The high degree of azimuthal symmetry allows finding the radial distribution of the electron density $\mathrm{n}_{e}(\mathrm{r})$ (Fig. 1c) by applying Abel inversion to the interferograms. The interferometry data also show the presence of a lower density plasma outside of the dense part of the jet. This halo plasma is formed due to the ablation of material from the foil at larger radii, and its electron density is considerably lower (by a factor of 3-5) than in the jet. The axial velocity of the tip of the jet estimated from the length of the jet seen in the XUV images, taking into account the time of the jet formation, gives jet tip velocity to $\mathrm{V}_{Z}>100 \mathrm{~km} / \mathrm{s}$. Direct measurements of the flow velocity were obtained using a Thomson scattering diagnostic (Harvey-Thompson et al. 2011). The measurements were performed at axial positions 12 and $20 \mathrm{~mm}$ above the foil at $\sim 410$ and $\sim 460$ ns after the current start respectively, when the tip of the jet 
reached a height of at least $30 \mathrm{~mm}$. The Thomson scattering operated in the collective scattering regime (scattering parameter $\alpha>1$ ) and measured the spectra of scattered light from several radial positions $(\sim 0.7 \mathrm{~mm}$ diameter each, separated by $1.3 \mathrm{~mm}$ ) across the jet and the halo plasma. The Doppler shift of the scattered spectrum corresponds to the flow velocity of $50 \mathrm{~km} / \mathrm{s}$ at $\mathrm{z}=12 \mathrm{~mm}$ and $60 \mathrm{~km} / \mathrm{s}$ for $\mathrm{z}=20 \mathrm{~mm}$. This is a factor $1.5-2$ smaller than velocity of the jet tip which at the time of the measurements reaches $\mathrm{z} \sim 35 \mathrm{~mm}$. The Thomson scattering measurements also show that the halo plasma surrounding the jet has the same velocity as the jet. The scattered spectrum is not only Doppler shifted, but is also broadened, especially for the radial positions corresponding to the dense part of the jet. The shapes of the spectra at these locations indicate the presence of ion acoustic features, which become distinguishable when the product of $\mathrm{ZT}_{e}$ (where $\mathrm{Z}$ is the mean ionization in the plasma and $\mathrm{T}_{e}$ is the electron temperature) is larger than the ion temperature $T_{i}$. Figure $2 \mathrm{a}$ shows the measured scattered spectrum on the jet axis compared with theoretical profiles calculated for several values of electron temperatures and the corresponding values of $\mathrm{Z}$ for these temperatures (assuming LTE). This comparison shows that the plasma electron temperature in the jet body is $\mathrm{T}_{e} \sim 15-20 \mathrm{eV}$ with $\mathrm{Z} \sim 4$. The velocity and temperature of the jet, measured by Thomson scattering, give an internal Mach number in the range $\mathrm{M}=$ $\mathrm{V}_{Z} / \mathrm{C}_{s} \sim 2.5-3$. A freely expanding flow with such internal Mach numbers should have relatively large half-opening angles $\phi=\tan ^{-1}(1 / \mathrm{M}) \sim 20^{\circ}$ for $\mathrm{M}=3$. The measured half-opening angle of the jet is much smaller $\left(\sim 2^{\circ}-5^{\circ}\right)$, corresponding to much larger internal Mach numbers $(\mathrm{M} \sim 10-30)$. This strongly suggests that the jet is not freely expanding, but that some confining force is present. The need for a confining force is also consistent with the observed slow increase of the jet diameter, with a radial velocity of $\mathrm{V}_{R} \sim 5 \mathrm{~km} / \mathrm{s}$, which is much smaller than the sound speed of $\mathrm{C}_{s} \sim 20 \mathrm{~km} / \mathrm{s}$ calculated from Thomson scattering measurements. The jet is surrounded by a low-density halo plasma which moves with the same velocity as the jet, but the thermal pressure of this plasma is not sufficient to explain the observed level of jet collimation. 3-D resistive MHD simulations of the jet formation show that the toroidal magnetic field diffuses through the heated foil and plays a role in the jet collimation. The $\mathrm{J} \times \mathrm{B}$ force associated with this toroidal magnetic field re-directs the flow of plasma ablated from the foil towards the axis, and the ram pressure of this converging flow is responsible for the collimation of the central, dense part of the jet.

The jet obtained and characterized in these experiments is supersonic, though the internal Mach number $(\mathrm{M} \sim 3)$ is smaller than typical internal Mach numbers in astrophysical jets $(\mathrm{M} \sim 20)$. The jet has a flow velocity $(50-100 \mathrm{~km} / \mathrm{s})$ comparable to those of the astrophysical jets $(\sim 200 \mathrm{~km} / \mathrm{s})$ and an aspect ratio (length/radius $\sim 20$ ) sufficient for designing a scaled jet-ambient interaction experiment under conditions when radiative cooling is playing a significant role. The first set of such experiments was performed using Ar ambient gas occupying all space above the foil (Suzuki-Vidal et al. 2012). In this case, both the jet formation and propagation were affected. To separate the jet formation and the interaction region, we recently started experiments where a cloud of ambient gas 

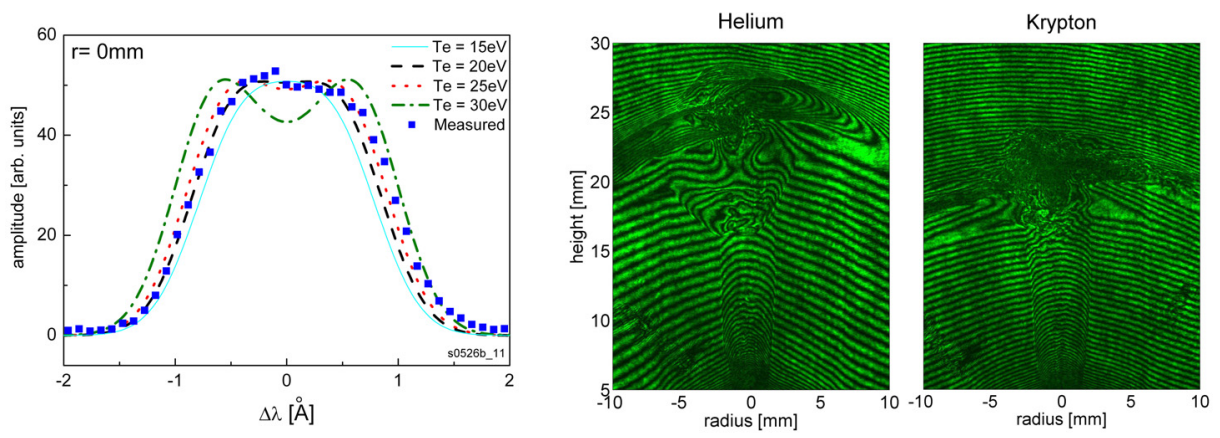

Fig. 2. Left: measured (dots) and theoretical Thomson scattering spectra. Right: laser interferograms showing interaction of the jet with ambient He and $\mathrm{Kr}$ gases of the same mass densities.

was formed at a distance $\sim 10-15 \mathrm{~mm}$ above the foil. Figure $2 \mathrm{~b}$ shows results of the first experiments with this set-up. Formation of the bow-shock is clearly seen, and the morphology of the interaction region strongly depends on the level of the radiative cooling, controlled by the choice of ambient material (e.g. He and $\mathrm{Kr}$ ).

This work was supported by the EPSRC Grant no. EP/G001324/1, by the NNSA under DOE Cooperative Agreements No. DE-F03-02NA00057 and No. DE-SC-0001063, by DOE SBIR Grant DE-FG02-08ER85030, and by a Marie Curie European Reintegration grant.

\section{References}

Bellan, P.M., You, S., \& Hsu, S.C., 2005, Ap\&SS, 298, 203

Ciardi, A., Lebedev, S.V., Frank, A., et al., 2007, Phys. Plasmas, 14, 056501

Ciardi, A., Lebedev, S.V., Frank, A., et al., 2009, ApJ, 691, L147

Foster, J.M., Wilde, B.H., Rosen, P.A., et al., 2005, ApJ, 634, L77

Gourdain, P.-A., Blesener, I.C., Greenly, J.B., et al., 2010, Phys. Plasmas, 17, 012706

Harvey-Thompson, A.J., Lebedev, S.V., et al., 2012, Phys. Rev. Lett., 106, 205002

Lebedev, S.V., Ciardi, A., Ampleford, D.J., et al., 2005, MNRAS, 361, 97

Loupias, B., Koenig, M., Falize, E., et al., 2007, Phys. Rev. Lett., 99, 265001

Remington, B.A., Drake, R.P., \& Ryutov, D.D., 2006, Rev. Mod. Phys., 78, 755

Suzuki-Vidal, Lebedev, S.V., et al., 2010, Phys. Plasmas, 17, 112708

Suzuki-Vidal, F., Bocchi, M., Lebedev, S.V., et al., 2012, Phys. Plasmas, 19, 022708 